\title{
Cellulose Nanofibers/Melanin Hybrid Aerogel Supported Phase Change Materials with Improved Photothermal Conversion Efficiency and Superior Energy Storage Density
}

Jiulong Chen ( $\square$ king12311231@gmail.com )

Sichuan University https://orcid.org/0000-0001-8921-3077

Yiyang Zhang

Sichuan University

Fugeng Wu

Sichuan University

Bin Guan

Sichuan University

Xiaosheng Du

Sichuan University https://orcid.org/0000-0001-8239-4303

Haibo Wang

Sichuan University

\section{Research Article}

Keywords: solar-thermal conversion, natural melanin, phase change materials, thermal energy storage

Posted Date: June 2nd, 2021

DOI: https://doi.org/10.21203/rs.3.rs-529394/v1

License: (c) (i) This work is licensed under a Creative Commons Attribution 4.0 International License.

Read Full License

Version of Record: A version of this preprint was published at Cellulose on August 21st, 2021. See the published version at https://doi.org/10.1007/s10570-021-04152-7. 


\section{Abstract}

Organic phase change materials (PCMs) have been widely applied in thermal energy storage fields due to their good structural stability, high energy storage density, adjustable phase change temperature and nontoxic. However, the poor solar-thermal conversion performance and structure stability restrict the largescale application of organic PCMs. Herein,novel PCM composites (CMPCMs) with good structure stability, improved photothermal conversion efficiency, and superior energy storage density were successfully synthesized by impregnating poly (ethylene glycol) (PEG) into cellulose nanofibers/melanin hybrid aerogel. The three-dimensional (3D) aerogel framework had good shape stability and strong encapsulation ability, which inhibited the leakage of PEG and enhanced the shape stability of the synthesized PCM. The differential scanning calorimetry (DSC) results showed that CMPCMs exhibited relatively high melting enthalpies ranging from 168.3 to $175.9 \mathrm{~J} / \mathrm{g}$, and the introduction of melanin almost unchanged the energy storage density of the synthesized PCM composites. Simulated sunlight test revealed that the introduction of melanin significantly improved the photothermal conversion efficiency of CMPCMs (from 47.2 to $85.9 \%$ ). The thermal cycling test and thermogravimetric analysis showed that CMPCMs possessed excellent thermal stability and good encapsulation ability. In conclusion, the synthesized CMPCMs showed great potential in the practical utilization and storage of solar energy.

\section{Introduction}

Solar energy is the most popular energy source because it is abundant, accessible, and inexhaustible. It has become an ideal choice for dealing with energy crises, fossil fuel shortages, and global warming. Currently, one of the most common applications of solar energy is solar thermal energy conversion. However, solar energy is not easy to store, and it is greatly affected by the change of day and night, which greatly limits the application of solar energy. Phase change materials (PCMs) currently have great application potential in the field of solar energy materials due to their ability to absorb or release a large amount of heat during the phase change process(Pielichowska and Pielichowski 2014). The application of phase change materials in solar thermal energy conversion can greatly improve energy utilization efficiency and overcome the problem of solar radiation interval. Organic solid-liquid phase change materials are widely used in thermal energy storage systems because of their excellent structural stability, excellent energy storage density, adjustable phase transition temperature, and non-toxic properties(Weigand and Fleischer 2014; Velasco-Carrasco et al. 2020). However, the large-scale application of PCMs in solar energy conversion is limited by the problem of liquid leakage during the phase change process(Pielichowska et al. 2015; Han et al. 2017; Woods et al. 2021). At the same time, the low solar-thermal conversion efficiency of organic phase change materials also limits the use of PCM in the field of solar energy conversion.

The main way to solve the problem of PCM leakage is to use microcapsules to wrap the PCM or use porous materials to adsorb the PCM, so that the shape stability of the PCM can be ensured during the phase change process and liquid leakage can be prevented. However, the above method introduces 
substances that cannot perform phase change energy storage into the PCM, resulting in a greatly reduced phase change enthalpy of the PCM. At present, an effective technology to prevent phase change liquid leakage due to high surface tension(WOIGNIER, T. and PHALIPPOU, J. 1989) and capillary force is to impregnate organic PCMs into cellulose-based aerogel(Hasnain 1998; Farid et al. 2004; Zhang et al. 2014; Ye et al. 2015). Aerogel is porous three-dimensional (3D) interconnect materials with extremely low density, ultra-high specific surface area and excellent insulating properties(Fricke 1988; Fricke and Tillotson 1997). However, since both cellulose-based aerogel and PCM are organic materials, they have the characteristics of poor solar-thermal conversion performance(Sharma et al. 2009; Yang et al. 2018), and the introduction of cellulose-based aerogel will reduce the phase change enthalpy of PCM, which greatly limits the application of PCM/cellulose aerogel composites in solar thermal conversion systems(Qian et al. 2019). Therefore, how to improve the solar-thermal conversion performance of the composite material and reduce the decrease in the PCM phase change enthalpy caused by the cellulosebased aerogel is an inevitable problem when preparing the PCM/cellulose aerogel composite(Sheng et al. 2019).

Melanin is the general term for a class of phenolic or indole biological macromolecular pigments with complex and diverse structures(Cordero and Casadevall 2020). Melanin is a very effective substance produced by organisms to fight ultraviolet rays(Meredith and Sarna 2006). When ultraviolet rays hit the melanin chemical chain, the melanin chemical chain vibrates at an extremely fast speed, converting $99 \%$ of the harmful ultraviolet rays into harmless heat(Forest and Simon 1998; Wakamatsu and Ito 2002; Ito and Wakamatsu 2003; Eisenman and Casadevall 2012; Wang et al. 2019). At the same time, the extraction of melanin from organisms is very simple and efficient(Di Mauro et al. 2017; Solano 2017), and does not require chemical synthesis(d'Ischia et al. 2013), so the use of natural melanin is more environmentally friendly. The introduction of melanin particles into PCM/cellulose aerogel composites can improve the thermal conversion efficiency of the composite material to sunlight, especially ultraviolet rays, and make composites more efficient(Brenner and Hearing 2008).

In this work, fresh cuttlefish ink was used as raw material to obtain natural melanin particles through centrifugal treatment and washing, and then cellulose nanofibers/melanin hybrid aerogel (CMA) based on melanin particles and cellulose nanofibers (CNF) were fabricated through induced by cation-induced gelation. After that, polyethylene glycol (PEG) was vacuum immersed into the aerogel to prepare the PCM composite material. The microstructure, loading rate, packaging performance, thermal stability and solarthermal conversion performance of composites are systematically studied. As expected, the 3D porous aerogel can fully support the PEG during the phase change process and prevent its leakage, and the addition of natural melanin particles can effectively improve the solar-thermal conversion efficiency of CMA/PEG composite PCMs (CMPCMs). At the same time, the reduction of the phase change enthalpy of the PCM is in a lower range. This means that CMPCMs have huge application potential in solar energy utilization systems.

\section{Materials And Methods}


CNF aqueous (length: 1-3 $\mu \mathrm{m}$, diameter: 4-10 nm, 1wt\%) was supplied from Guilin Qihong Tech Co., Ltd., China. Cuttlefish ink (Unprocessed, food grade) was purchased from Qingdao Xinjie Aquatic Products Co., Ltd., China. Aluminum nitrate nonahydrate $\left(\mathrm{Al}\left(\mathrm{NO}_{3}\right)_{3} \cdot 9 \mathrm{H}_{2} \mathrm{O}, \mathrm{AR}\right)$ was obtained from Chengdu Kelong Chemical Reagent Co., Ltd., China. PEG was provided by Chengdu Chron Chemicals Co., Ltd., China.

Extraction of natural melanin

Firstly, use centrifugation (12000 rpm, $20 \mathrm{~min}$ ) to separate melanin particles from fresh cuttlefish ink. Then, clean melanin particles were received after 4-times of centrifuge-after-washing process with deionized water. After Freeze-drying $\left(-50^{\circ} \mathrm{C}, 20 \mathrm{~Pa}\right)$ for $48 \mathrm{~h}$, excess water was removed, and dry melanin particles were obtained.

Preparation of CMAs

Firstly, prepare the $\mathrm{Al}\left(\mathrm{NO}_{3}\right)_{3} / \mathrm{H}_{2} \mathrm{O}$ solution with a concentration of $1.84 \mathrm{wt} \%$ ( $50 \mathrm{mmol} / \mathrm{L}$ ) by dissolving $3.75 \mathrm{~g} \mathrm{Al}\left(\mathrm{NO}_{3}\right)_{3} \cdot 9 \mathrm{H}_{2} \mathrm{O}$ into $200 \mathrm{~mL}$ deionized water. Then add a certain amount of melanin into $\mathrm{CNF}$ (The mass ratio of CNF to melanin is 4:1, 2:1, 4:3,1:1), and mechanical stirring for 15 min in a container. Next, add $15 \mathrm{~mL} \mathrm{Al}\left(\mathrm{NO}_{3}\right)_{3} / \mathrm{H}_{2} \mathrm{O}$ solution (The concentration is $1.84 \mathrm{wt} \%$ ) into the container. After 1 day, the $\mathrm{Al}\left(\mathrm{NO}_{3}\right)_{3} / \mathrm{H}_{2} \mathrm{O}$ solution was changed 1 time/day for 2 days. Then, aerogel was transferred in to freeze-dry machine for $48 \mathrm{~h}$ to obtain dried CMAs, respectively labeled as CMA-1, CMA-2, CMA-3, and CMA-4. At the same time, for the convenience of experimental control, a sample without melanin was also prepared by a completely consistent method, and it was named CA.

\section{Fabrication of CMPCMs}

Firstly, PEG was heated to $85^{\circ} \mathrm{C}$ in the vacuum oven. Then, the CA and CMAs were placed in PEG that turns into liquid phase. After that, the bubbles in the aerogel was removed by increasing the vacuum until the oven nearly completely vacuums and continued to $1 \mathrm{~h}$. Then, the CA/PEG composite PCM (CPCM) and CMPCMs were prepared, as presented in Scheme 1. Respectively marked as CPCM, CMPCM-1, CMPCM-2, CMPCM-3, and CMPCM-4.

Characterization

The chemical structure of melanin was analyzed by a Fourier transform infrared spectrometer (FTIR, PE Spectrum Two, PerkinElmer, America), the spectra were from 648 to $4000 \mathrm{~cm}^{-1}$, with a resolution of 2 $\mathrm{cm}^{-1}$. A Lambda 750 spectrophotometer (Perkin Elmer, America) was used to record the UV-vis-NIR absorption spectrum of the natural melanin particle.

The chemical structure of CMAs and CMPCMs was characterized by an X-ray photoelectron spectroscopy (XPS) spectrometer (K-Alpha+, Thermo Scientific, America). 
The internal morphologies and structures of CA, CMAs, CPCM, and CMPCMs were observed by a scanning electron microscope (SEM, Helios G4 UC, Thermo Scientific, America).

The phase transition performance of the CPCM and CMPCMs was measured by differential scanning calorimetry (DSC, 214 Polyma, Netzsch, Germany) with temperature range from 15 to $85^{\circ} \mathrm{C}$ at a heating/freezing rate of $10{ }^{\circ} \mathrm{C} \mathrm{min}^{-1}$ under nitrogen atmosphere. The thermal stability of the CPCM and CMPCMs was evaluated by a TG analyzer (209 F3 Tarsus, Netzsch, Germany), with temperature range from 50 to $800{ }^{\circ} \mathrm{C}$ at a heating rate $10^{\circ} \mathrm{C} \mathrm{min}^{-1}$ under nitrogen atmosphere.

A solar-thermal conversion measurement system was used to evaluate the thermal conversion and storage capability of the CPCM and CMPCMs in practical application, as present in Scheme 2. To simulated sunlight, a solar simulator (CHF-XM500, Beijing Changtuo, China) was used. To measure and record the temperature variations of $\mathrm{CPCM}$ and $\mathrm{CMPCM}$ during the light test, a thermocouple thermometer (F-8855, Shenzhen Flank Electronics Co., Ltd, China) was used. The CPCM and CMPCMs were placed under a constant irradiation intensity of $250 \mathrm{~mW} \mathrm{~cm}^{-2}$, and the thermocouple was placed into the center of the CPCM and CMPCMs. The solar-thermal conversion and storage efficiency $(\varepsilon)$ of CMPCMs was calculated using the equation:

$\varepsilon=\frac{m \Delta H_{m}}{I S\left(T_{e}-T_{s}\right)} \times 100 \%$

where $m, S$, and $\Delta H_{m}$ represent the weight, received light irradiation area, and melting enthalpy of the CMPCM sample, respectively, / represents irradiation intensity, and $T_{s}$ and $T_{e}$ represent the start and end times of phase transition of CMPCMs, respectively. To intuitively demonstrate the leakage, prevent property of $\mathrm{CPCM}$ and $\mathrm{CMPCMs}$, the samples were heated at $80^{\circ} \mathrm{C}$ and photographed at $0 \mathrm{~min}, 10 \mathrm{~min}, 20$ $\min$ and $30 \mathrm{~min}$.

\section{Results And Discussion}

\section{Characterization of melanin}

FTIR test is used to identify the chemical structure of melanin, the FITR spectra result is shown in Fig. 1a. The strong resonance absorption peak at $3404 \mathrm{~cm}^{-1}$ corresponds to the $\mathrm{N}-\mathrm{H}$ stretching vibration of indole and $\mathrm{O}-\mathrm{H}$ group, indicating that it has a typical melanin indole structure. A peak at $2922 \mathrm{~cm}^{-1}$ corresponds to the stretching vibration of aliphatic $\mathrm{C}-\mathrm{H}$ groups, shows that cuttlefish melanin has aromatic hydrocarbon structure. This weak peak at $2922 \mathrm{~cm}^{-1}$ is one of the characteristics of cuttlefish melanin FTIR spectra. The characteristic strong band at $1616 \mathrm{~cm}^{-1}$ is attributed to the $\mathrm{C}=0$ stretching vibration and the $\mathrm{C}=\mathrm{C}$ vibration of the aromatic ring or the symmetric stretching of the COO-group. This band together with the strong absorption peak at $3400 \mathrm{~cm}^{-1}$ indicates the existence of $\mathrm{COOH}$ structure. Therefore, it can be concluded that squid black pigments contain specific binding sites $(\mathrm{O}-\mathrm{H}, \mathrm{N}-\mathrm{H}$ and $\mathrm{COOH}$ ) of melanin. The UV-visible absorption spectrum (Fig. 1b) indicates that the sample melanin image 
has no obvious chromogenic peak and has monotonic broad-band absorbance in the ultraviolet to visible wavelength range.

\section{Chemical structure of CMAs and CMPCMs}

XPS was used to analysis the element composition of CPCM, CMPCM-2 and CMPCM-4. Compared with the pure CPCM sample, the spectrum of CMPCM clearly shows the presence of nitrogen (Table 1). XPS spectrums obtained from pure CPCM samples (Fig. 2a) showed signals near 532eV and $286.0 \mathrm{eV}$, indicating the presence of oxygen and carbon, respectively. There is also a weak peak at $400 \mathrm{eV}$, which is presumed to be nitrogen-containing impurities. The XPS spectrums of CMPCM-2 (Fig. 2b) and CMPCM-4 (Fig. 2c) also showed strong peaks around $532 \mathrm{eV}$ and $286.0 \mathrm{eV}$. A new peak appeared on the right side of the impurity peak of $400 \mathrm{eV}$, and the new peak was more obvious with the increase of melanin content. This indicates that CMPCM is composed of carbon, nitrogen and oxygen, and nitrogen is introduced by the doping of melanin. The analysis of XPS data showed that the addition of melanin increased the nitrogen content of the sample, which was caused by the nitrogen-containing functional groups of melanin.

As shown in Fig. 2d, the high-resolution XPS $\mathrm{C}$ 1s spectrum of CPCM shows that the carbon peak consists of C-C $(284.8 \mathrm{eV}), \mathrm{C}-0(286.0 \mathrm{eV})$ and C $=0(288.0 \mathrm{eV})$. The high-resolution XPS C $1 \mathrm{~s}$ spectra of CMPCM-2 (Fig. 2e) and CMPCM-4 (Fig. 2f) showed that the signal of carbon peak was contributed by C-C (284.8eV/284.7eV), C-O (286.4eV/286.0eV), C = O (287.8eV/288.5eV) and C-N/N-H (286.2eV). The high resolution XPS N 1 spectra showed that both CMPCM-2 (Fig. 2g) and CMPCM-4 (Fig. 2h) contained N-H $(399.6 \mathrm{eV})$ and $-\mathrm{NH}^{+}-(400.3 \mathrm{eV})$ functional groups. The high resolution XPS 01 s spectrum of CMPCM-4 (Fig. 2i) corresponds to $\mathrm{C}-\mathrm{O}(532.2 \mathrm{eV})$ and $\mathrm{C}=\mathrm{O}(532.8 \mathrm{eV})$, respectively. The results of XPS further demonstrated that the in-situ polymerization of melanin and CPCM was successful.

Table 1

XPS analysis of the CPCM and CMPCMs

\begin{tabular}{|llll|}
\hline Element & CPCM & CMPCM-2 & CMPMCM-4 \\
\hline C (\%) & 40.8 & 53.21 & 53.04 \\
\hline O (\%) & 57.54 & 43.69 & 40.39 \\
\hline N (\%) & 1.65 & 3.11 & 6.57 \\
\hline
\end{tabular}

Morphology of CMAs and CMPCMs

The microstructures of CA, CMAs, CPCM and CMPCMs were studied by SEM, as presented in Fig. 3. CA (Fig. 3a) is porous under microscope, most of the pore sizes are in the range of a few microns to a few hundred microns. The structure of pore wall is nano sheet, and the nano sheet has a certain sense of hierarchy. CMAs (Fig. 3b is CMA-2 and Fig. 3c is CMA-4) are also porous under the microscope, and the pore size is mostly in the range of a few microns to a few hundred microns. The main framework is still 
CA structure. The only difference is that there are irregular protrusions on the pore wall structure, which are formed by the addition of melanin.

The composite materials CPCM (Fig. 3d) and CMPCMs (Fig. 3e is CMPCM-2 and Fig. $3 f$ is CMPCM-4) are blocky structures under the microscope. PEG fills the whole pore structure and forms blocky structure. It still can see the CA framework in some angle sections (Fig. 3d and Fig. 3f), which is enough to support the judgment. The formation of blocky structure indicates that PEG and CA and CMAs have good compatibility. The porous structure and good compatibility can make the low-density aerogel encapsulate a large number of PEG and maintain a higher energy storage density.

Shape stability of CMPCMs

Shape ability is a very important property of composite phase change materials, which often plays a decisive role in the practical application of such materials. When the temperature is higher than the phase transition temperature, most organic PCMs will show fluidity and leak out from the composites, which is the leakage problem of organic phase change materials. To solve this problem, inorganic or organic carrier materials are often used to encapsulate organic PCMs, so it is necessary to measure the encapsulation ability of the materials. The encapsulation ability can be judged by detecting the leakage of materials at different time in the phase transformation stage. For CPCM and CMPCMs, the leakage material in the phase transformation stage is PEG. In this experiment, the phase transition temperature is $80^{\circ} \mathrm{C}$, and the conditions of $0 \mathrm{~min}, 10 \mathrm{~min}, 20 \mathrm{~min}$ and $30 \mathrm{~min}$ are recorded. The comparison of test results is shown in Fig. 4.

At $25{ }^{\circ} \mathrm{C}, \mathrm{PEG}, \mathrm{CPCM}$ and CMPCM-4 were smooth white opaque solid, rough white opaque solid and rough black opaque solid, respectively. After heating at $80^{\circ} \mathrm{C}$ for 10 minutes, PEG began to melt, and the filter paper was wet. CPCM and CMPCM-4 did not change significantly, and the filter paper remained dry. After heating at $80{ }^{\circ} \mathrm{C}$ for 20 minutes, PEG melts obviously and cannot keep its original shape, the filter paper is wetter. CPCM and CMPCM-4 began to leakage some liquid, and the filter paper was slightly wet. After heating at $80^{\circ} \mathrm{C}$ for 30 minutes, a large amount of PEG melts and becomes semi solid and semi liquid, and liquid begins to accumulate on the filter paper. CPCM and CMPCM-4 slightly leakage, the morphology remained unchanged, the filter paper is slightly wet. In general, the difference between CPCM and CMPCMs is not obvious, but compared with pure PEG, it has stronger thermal stability and better encapsulation ability.

Thermal properties of CMPCMs

Thermal properties of materials are the different thermal physical properties of materials in the process of using at different temperatures and conditions. Heat capacity is one of the most important thermal properties of phase change energy storage materials. Differential scanning calorimetry (DSC) can measure the specific heat capacity and related thermal properties of samples by measuring and analyzing the data of heat flow corresponding to time or temperature input to the samples. The heat energy storage ability has a very strong connection between phase change properties. Therefore, in order 
to quantitatively evaluate the effect of the added melanin particles on the PCM phase change enthalpy, DSC analysis was used. Processed the result with software, the melting enthalpy $\left(\Delta H_{m}\right)$, crystallization enthalpy $\left(\Delta H_{c}\right)$, melting and crystallization point $\left(T_{m}\right.$ and $\left.T_{c}\right)$ were collected in Table 2 , and the DSC curves of pure PEG, CPCM and CMPCMs were presented in Fig. 5. As expected, a separate and obvious peak could be observed in both heating and cooling processes for all samples. Moreover, the DSC curves exhibited the endothermic peaks of CPCM and CMPCMs in the range of $66.3-69.8^{\circ} \mathrm{C}$ and the exothermic peaks in the range of $34.7-38.1{ }^{\circ} \mathrm{C}$, which were almost the same as pure PEG. The reason is that the melanin particles are only dispersed in the aerogel, and there is no chemical reaction between the PEG and the melanin particles, so the melanin particles will not affect the heat absorption capacity of the PEG. But with the increase of melanin content, the peak of crystallization slightly moves to low temperature, and the peak of melting slightly moves to high temperature. This arises because other substance restricted arrangement of PEG molecules and effect their phase transition process. This effect will hinder the crystallization and melting process of the PEG.

\begin{tabular}{|lllll|}
\hline \multicolumn{5}{|c|}{ Table 2 Thermal storage property of PEG, CPCM and CMPCMs } \\
\hline Sample & $T_{m}\left({ }^{\circ} \mathrm{C}\right)$ & $\Delta H_{m}(\mathrm{~J} / \mathrm{g})$ & $T_{c}\left({ }^{\circ} \mathrm{C}\right)$ & $\Delta H_{c}(\mathrm{~J} / \mathrm{g})$ \\
\hline PEG & 65.9 & 197.6 & 33.9 & 178.4 \\
\hline CPCM & 69.8 & 176.3 & 34.7 & 165.9 \\
\hline CMPCM-1 & 69.1 & 175.9 & 37.2 & 164.6 \\
\hline CMPCM-2 & 66.3 & 173.6 & 36.0 & 163.5 \\
\hline CMPCM-3 & 68.0 & 167.1 & 37.6 & 160.1 \\
\hline CMPCM-4 & 67.9 & 168.3 & 38.1 & 161.8 \\
\hline
\end{tabular}

As shown in Table 2, the $\Delta H_{m}$ of pure PEG, CPCM and CMPCMs were $197.6 \mathrm{~J} / \mathrm{g}, 176.3 \mathrm{~J} / \mathrm{g}, 175.9 \mathrm{~J} / \mathrm{g}$, $173.6 \mathrm{~J} / \mathrm{g}, 167.1 \mathrm{~J} / \mathrm{g}, 168.3 \mathrm{~J} / \mathrm{g}$, respectively and $\Delta H_{c}$ were $178.4 \mathrm{~J} / \mathrm{g}, 165.9 \mathrm{~J} / \mathrm{g}, 164.6 \mathrm{~J} / \mathrm{g}, 163.5 \mathrm{~J} / \mathrm{g}$, $160.1 \mathrm{~J} / \mathrm{g}, 161.8$, respectively. It can be found that melting enthalpy of CPCM is about $11 \%$ lower than that of PEG and crystallization enthalpy of CPCM is about 7\% lower than of PEG, because CNF and other substances will replace a certain amount of PEG with the same quality, and the overall enthalpy is reduced. With the increase of melanin content, the enthalpy of CMPCMs decreases gradually, which indicates that melanin can also replace PEG, which has a negative impact on the overall energy storage properties of the sample. This also shows that the porous structure of CNF aerogel has good bearing capacity for PEG.

Thermal reliability can reflect the performance changes of materials in long-term use. For phase change energy storage materials, their energy storage performance will generally change with the increase of use times, and the change of performance determines how to apply the materials. The thermal energy storage properties of the sample after 100 times of heating and cooling from $0{ }^{\circ} \mathrm{C}$ to $80^{\circ} \mathrm{C}$ are shown in the 
Table 3. The melting point and crystallization point of CPCM and CPMCMs samples had negligible changes after 100 repeated thermal cycles. Compared with samples before experiment, the melting point and crystallization point of CPCM and CPMCMs samples decreased by about $2 \%$ respectively. The results of accelerated thermal cycling measurement show that CPCM and CPMCMs have excellent thermal reliability.

Table 3

Thermal storage property of CPCM and CMPCMs after thermal cycling

\begin{tabular}{|lllll|}
\hline Sample & $T_{m}\left({ }^{\circ} \mathrm{C}\right)$ & $\Delta H_{m}(\mathrm{~J} / \mathrm{g})$ & $T_{c}\left({ }^{\circ} \mathrm{C}\right)$ & $\Delta H_{c}(\mathrm{~J} / \mathrm{g})$ \\
\hline СРCM & 69.8 & 171.9 & 34.6 & 162.1 \\
\hline СMPCM-1 & 69.2 & 172.8 & 37.2 & 162.2 \\
\hline CMPCM-2 & 66.4 & 169.6 & 36.1 & 160.4 \\
\hline СМРCM-3 & 68.0 & 165.1 & 37.5 & 157.8 \\
\hline СМРCM-4 & 67.9 & 165.7 & 38.0 & 158.9 \\
\hline
\end{tabular}

Thermal stability of CMPCMs

Thermal stability refers to ability of a material to remain stable or resist thermal shock at a certain temperature. Thermogravimetry (TG) is one kind of thermal analysis method to measure the relationship between the mass of sample and temperature under temperature-control program. By thermogravimetry, the stability of the samples under different temperature conditions can be summarized, the temperature range suitable for the normal operation of different samples can be found, and the change of internal structure of different samples can be inferred.

Figure 6 displays the TG graphs of CPCM and CMPCMs. The TG curves of CPCM and CMPCMs are similar, almost all the curves have the characteristics of one-step thermal decomposition, and their detailed $T_{\max }$ (maximum weight loss rate temperature), $T_{-50 \mathrm{wt}}$ (50 wt\% weight loss temperature) and char residue at $650^{\circ} \mathrm{C}$ are summarised in Table 4 . The decomposition temperature is between $350{ }^{\circ} \mathrm{C}$ and $420^{\circ} \mathrm{C}$. It shows that melanin has no chemical reaction with PEG, and PEG will volatilize in this temperature range. The maximum mass loss rate is about $392{ }^{\circ} \mathrm{C}$. With the increase of melanin, the curve will gradually slope downward at $150{ }^{\circ} \mathrm{C}$, which means melanin will volatilize from $150^{\circ} \mathrm{C}$ to $350^{\circ} \mathrm{C}$. The residual weight of each sample is different, which shows melanin can affect the structure of the material, but the distribution of the influence is not uniform. With different structure, the sample will have different residual weight. 
Table 4

Thermal stability properties of the CPCM and CMPCMs

\begin{tabular}{|llll|}
\hline Sample & $T_{\text {-50 } w t \%}$ & $T_{\max }$ & char yield at $650{ }^{\circ} \mathrm{C}(w t \%)$ \\
& $\left({ }^{\circ} \mathrm{C}\right)$ & $\left({ }^{\circ} \mathrm{C}\right)$ & \\
\hline CPCM & 395.8 & 422.3 & 2.71 \\
\hline CMPCM-1 & 393.2 & 418.6 & 13.86 \\
\hline CMPCM-2 & 390.2 & 423.8 & 0.57 \\
\hline CMPCM-3 & 391.3 & 417.8 & 7.54 \\
\hline CMPCM-4 & 391.4 & 418.7 & 9.69 \\
\hline
\end{tabular}

Solar-thermal conversion and storage of CMPCMs

Photothermal conversion efficiency reflects the material's ability to use sunlight, which is an important property of photothermal materials. The higher the photothermal conversion efficiency is, the better the thermal storage performance of the composite is, and the practical application is more extensive. In order to test the photothermal conversion and thermal storage capacity of CMPCMs under practical conditions, we carried out simulated sunlight experiments on CPCM and CMPCMs. A cylindrical sample with a diameter of $3 \mathrm{~cm}$ and a height of $1 \mathrm{~cm}$ is placed in an insulating foam box and exposed directly to the 250 $\mathrm{mW} \mathrm{cm}{ }^{-2}$ irradiance simulator. Thermocouples are used to measure the temperature of CMPCM over time. A data recorder is connected to collect the temperature of CMPCMs. As shown in scheme 2.

Figure 7 shows the temperature-time curve of CMPCMs under illumination. The temperature of the sample rises gradually from the time of irradiating the sample. When the temperature of each sample rises to the range of $50-55^{\circ} \mathrm{C}$, the heating curve begins to slow down, and the heating rate drops sharply. This change is due to the endothermic process of PEG phase transition in CMPCMs. After the phase transition, the temperature of the sample continues to rise, and when the measured temperature reaches $80^{\circ} \mathrm{C}$, the illumination was stopped immediately. After illumination stopped, the actual sample temperature continued to rise to $83^{\circ} \mathrm{C}$, and then began to cool down. During the cooling process, the solidification temperature of PEG is about $50^{\circ} \mathrm{C}$. From the image, we can see that with the increase of the mass ratio of $\mathrm{CNF}$ and melanin, the phase transition time becomes shorter.

After calculation, the photothermal conversion and storage efficiency of CPCM, CMPCM-1, CMPCM-2, CMPCM-3, and CMPCM- 4 are $47.2 \%, 61.4 \%, 70.8 \%, 78.6 \%$ and $85.9 \%$ respectively. The results show that $\mathrm{CPCM}$ after adding melanin can obtain the photothermal properties of melanin, and the photothermal conversion efficiency increases with the increase of the proportion of melanin. Compared with CPCM, the photothermal conversion rates of MPCM-1, MPCM-2, MCM-3, and MPCM-4 are increased by $30.1 \%$, $50.0 \%, 66.5 \%$ and $82.0 \%$, respectively, which can show that melanin can greatly improve the photothermal properties of CPCM and optimize it to a phase change material with high photothermal properties and high energy storage density. 


\section{Conclusions}

Novel CMPCMs with good structure stability, improved photothermal conversion efficiency, and superior energy storage density were successfully synthesized by impregnating PEG into cellulose nanofibers/melanin hybrid aerogel which was prepared by in situ polymerization method. The 3D aerogel framework had good shape stability and strong encapsulation ability, which inhibited the leakage of PEG and enhanced the shape stability of the synthesized PCM. The DSC results showed that CMPCMs exhibited relatively high melting enthalpies ranging from 168.3 to $175.9 \mathrm{~J} / \mathrm{g}$, and the introduction of melanin almost unchanged the energy storage density of the synthesized PCM composites. Simulated sunlight test revealed that the introduction of melanin significantly improved the photothermal conversion efficiency of CMPCMs (from 47.2 to $85.9 \%$ ). The thermal cycling test and thermogravimetric analysis showed that CMPCMs possessed excellent thermal stability and good encapsulation ability. In conclusion, the synthesized CMPCMs showed great potential in the practical utilization and storage of solar energy.

\section{Declarations}

Competing of interest The authors declare that they have no known competing financial interests or personal relationships that could have appeared to influence the work reported in this paper.

Ethical approval The article does not include human participants and/or animals research.

Authorship contribution Jiulong Chen: Investigation, Writing - Original Draft, Writing - Review \& Editing, Visualization. Yiyang Zhang: Data curation, Formal analysis. Fugeng Wu: Validation. Bin Guan: Software. Xiaosheng Du: Conceptualization, Methodology, Project administration, Resources. Haibo Wang: Supervision, Funding acquisition.

Acknowledgements This work was funded by the National Natural Science Foundation of China (NO. 51773129, 51903167). The author appreciates Professor Xinfeng Quan from Sichuan University Pittsburgh Institute for his careful guidance. Also thank Yulong Tan, Jianing Xu from College of Biomass Science and Engineering of Sichuan University and Jinbo Zhao from Sichuan University - Pittsburgh Institute for their help in the experiment.

\section{References}

1. Brenner M, Hearing VJ (2008) The Protective Role of Melanin Against UV Damage in Human Skin. Photochem Photobiol 84:539-49

2. Cordero RJB, Casadevall A (2020) Melanin. Curr Biol 30:R142-R143. https://doi.org/10.1016/j.cub.2019.12.042

3. d'Ischia M, Wakamatsu K, Napolitano A, et al (2013) Melanins and melanogenesis: methods, standards, protocols. Pigment Cell Melanoma Res 26:616-633. https://doi.org/10.1111/pcmr.12121 
4. Di Mauro E, Xu R, Soliveri G, Santato C (2017) Natural melanin pigments and their interfaces with metal ions and oxides: emerging concepts and technologies. MRS Commun 7:141-151. https://doi.org/10.1557/mrc.2017.33

5. Eisenman HC, Casadevall A (2012) Synthesis and assembly of fungal melanin. Appl Microbiol Biotechnol 93:931-940. https://doi.org/10.1007/s00253-011-3777-2

6. Farid MM, Khudhair AM, Razack SAK, Al-Hallaj S (2004) A review on phase change energy storage: materials and applications. Energy Convers Manag 45:1597-1615. https://doi.org/10.1016/j.enconman.2003.09.015

7. Forest SE, Simon JD (1998) Wavelength-dependent Photoacoustic Calorimetry Study of Melanin. Photochem Photobiol 68:296-298. https://doi.org/10.1111/j.1751-1097.1998.tb09684.x

8. Fricke $J$ (1988) Aerogels - highly tenuous solids with fascinating properties. Glas Glass Ceram Gels 100:169-173. https://doi.org/10.1016/0022-3093(88)90014-2

9. Fricke J, Tillotson T (1997) Aerogels: Production, characterization, and applications. Thin Solid Films 297:212-223. https://doi.org/10.1016/S0040-6090(96)09441-2

10. Han GGD, Li H, Grossman JC (2017) Optically-controlled long-term storage and release of thermal energy in phase-change materials. Nat Commun 8:1446. https://doi.org/10.1038/s41467-017-01608y

11. Hasnain SM (1998) Review on sustainable thermal energy storage technologies, Part I: heat storage materials and techniques. Energy Convers Manag 39:1127-1138. https://doi.org/10.1016/S01968904(98)00025-9

12. Ito S, Wakamatsu K (2003) Quantitative Analysis of Eumelanin and Pheomelanin in Humans, Mice, and Other Animals: a Comparative Review. Pigment Cell Res 16:523-531. https://doi.org/10.1034/j.1600-0749.2003.00072.x

13. Meredith P, Sarna T (2006) The physical and chemical properties of eumelanin. Pigment Cell Res 19:572-594. https://doi.org/10.1111/j.1600-0749.2006.00345.x

14. Pielichowska K, Pielichowski K (2014) Phase change materials for thermal energy storage. Prog Mater Sci 65:67-123. https://doi.org/10.1016/j.pmatsci.2014.03.005

15. Pielichowska K, Szatkowski P, Zambrzycki M, Macherzynska B (2015) Polyurethane/graphene nanocomposites as phase change materials for thermal energy storage. In: 2015 IEEE 15th International Conference on Nanotechnology (IEEE-NANO). IEEE, Rome, pp 105-108

16. Qian Y, Han N, Zhang Z, et al (2019) Enhanced Thermal-to-Flexible Phase Change Materials Based on Cellulose/Modified Graphene Composites for Thermal Management of Solar Energy. ACS Appl Mater Interfaces 11:45832-45843. https://doi.org/10.1021/acsami.9b18543

17. Sharma A, Tyagi VV, Chen CR, Buddhi D (2009) Review on thermal energy storage with phase change materials and applications. Renew Sustain Energy Rev 13:318-345.

https://doi.org/10.1016/j.rser.2007.10.005

18. Sheng N, Nomura T, Zhu C, et al (2019) Cotton-derived carbon sponge as support for form-stabilized composite phase change materials with enhanced thermal conductivity. Sol Energy Mater Sol Cells 
192:8-15. https://doi.org/10.1016/j.solmat.2018.12.018

19. Solano F (2017) Melanin and Melanin-Related Polymers as Materials with Biomedical and Biotechnological Applications-Cuttlefish Ink and Mussel Foot Proteins as Inspired Biomolecules. Int J Mol Sci 18:1561. https://doi.org/10.3390/ijms18071561

20. Velasco-Carrasco M, Chen Z, Aguilar-Santana JL, Riffat S (2020) Experimental Evaluation of Phase Change Material Blister Panels for Building Application. Future Cities Environ 6:6. https://doi.org/10.5334/fce.84

21. Wakamatsu K, Ito S (2002) Advanced Chemical Methods in Melanin Determination. Pigment Cell Res 10

22. Wang K, Hou Y, Poudel B, et al (2019) Melanin-Perovskite Composites for Photothermal Conversion. Adv Energy Mater 9:1901753. https://doi.org/10.1002/aenm.201901753

23. Weigand R, Fleischer AS (2014) The influence of nanoparticle loading and surfactant on the viscosity of nanoenhanced energy storage materials. In: Fourteenth Intersociety Conference on Thermal and Thermomechanical Phenomena in Electronic Systems (ITherm). IEEE, Orlando, FL, USA, pp 846-850

24. WOIGNIER, T., PHALIPPOU, J. (1989) SCALING LAW VARIATION OF THE MECHANICAL PROPERTIES OF SILICA AEROGELS. J Phys Colloq 50:C4-179-C4-184. https://doi.org/10.1051/jphyscol:1989429

25. Woods J, Mahvi A, Goyal A, et al (2021) Rate capability and Ragone plots for phase change thermal energy storage. Nat Energy 6:295-302. https://doi.org/10.1038/s41560-021-00778-w

26. Yang J, Li X, Han S, et al (2018) High-quality graphene aerogels for thermally conductive phase change composites with excellent shape stability. J Mater Chem A 6:5880-5886. https://doi.org/10.1039/C8TA00078F

27. Ye S, Zhang Q, Hu D, Feng J (2015) Core-shell-like structured graphene aerogel encapsulating paraffin: shape-stable phase change material for thermal energy storage. J Mater Chem A 3:40184025. https://doi.org/10.1039/C4TA05448B

28. Zhang Y, Zheng X, Wang H, Du Q (2014) Encapsulated phase change materials stabilized by modified graphene oxide. J Mater Chem A 2:5304-5314. https://doi.org/10.1039/C3TA15242A

\section{Figures}



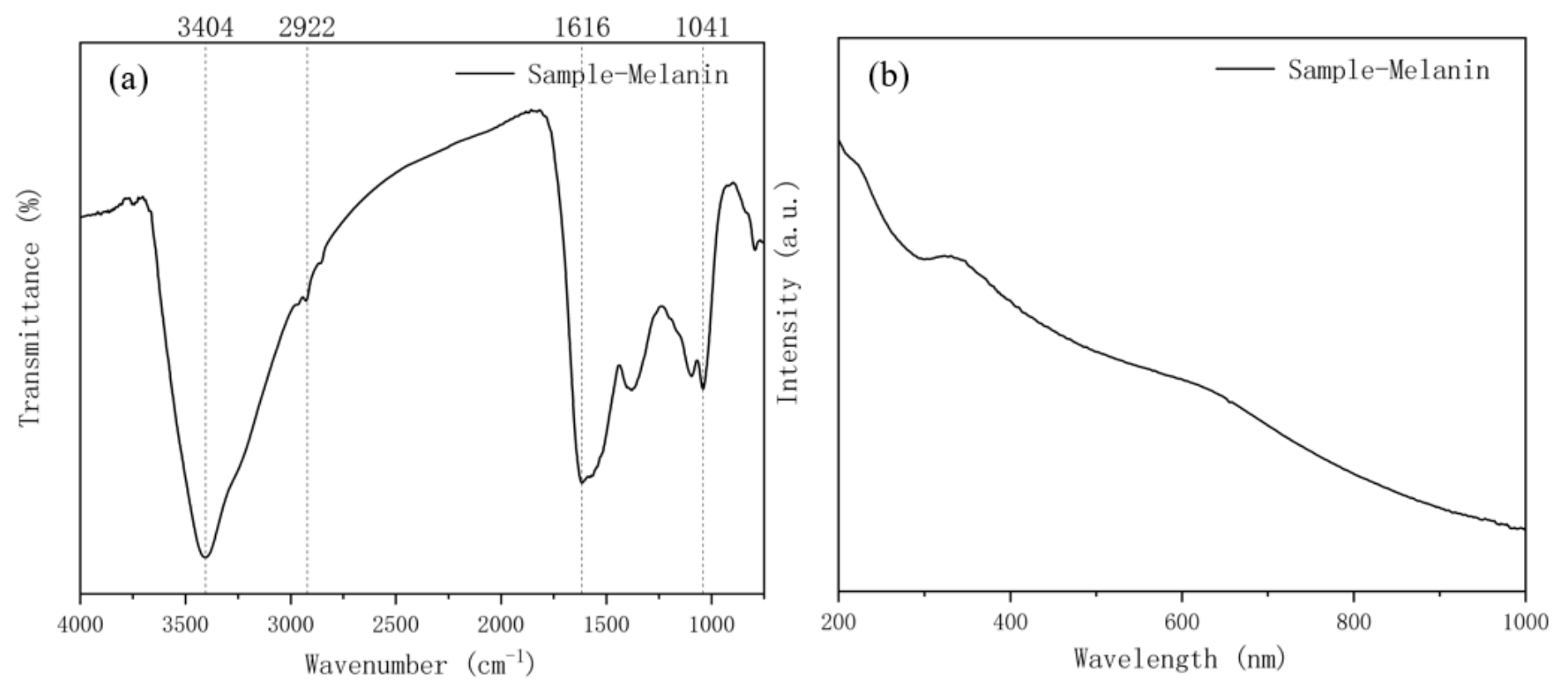

Figure 1

Characterization of melanin. (a) FITR spectra, (b) UV-vis-NIR absorption spectrum. 

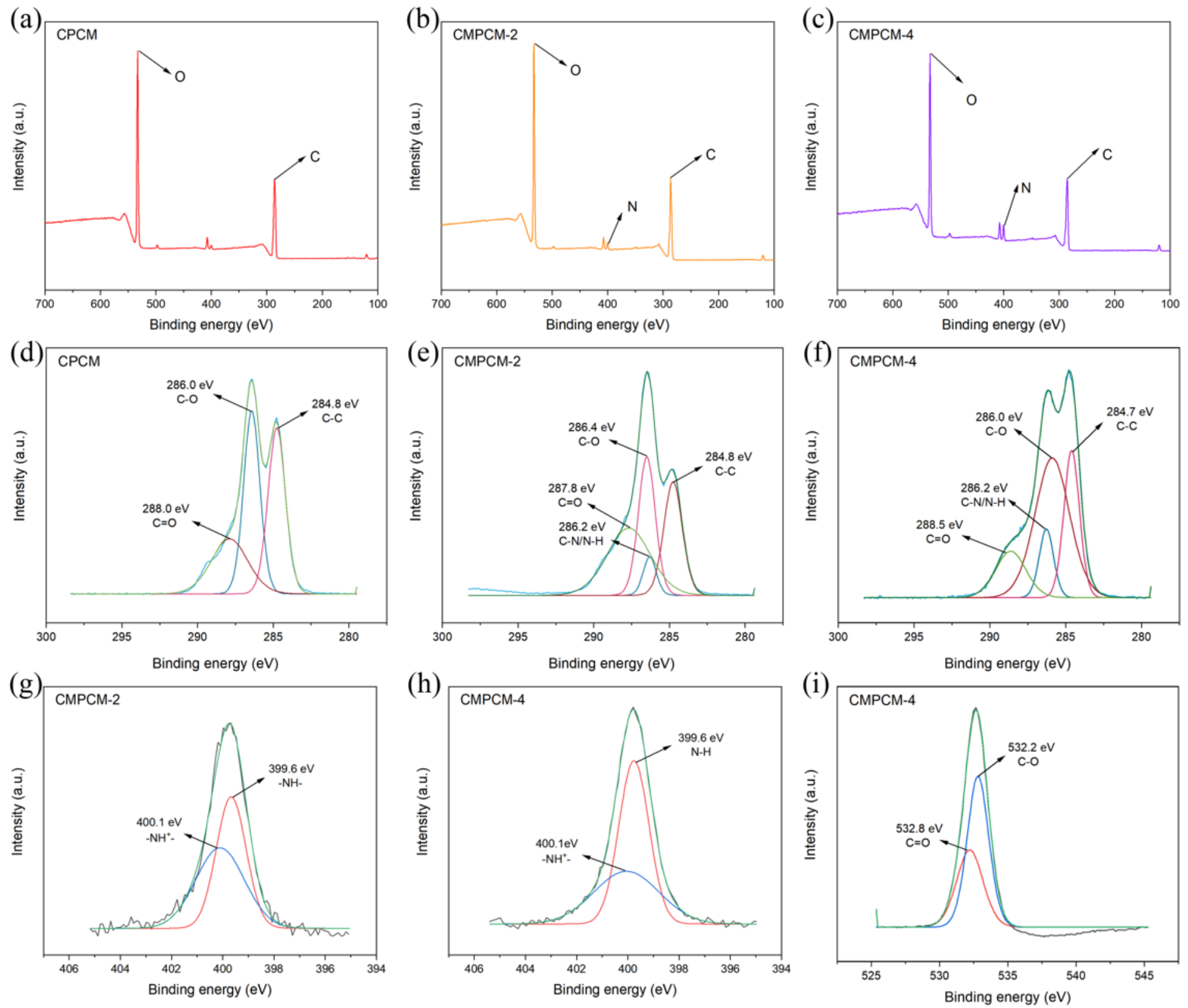

Figure 2

The XPS spectra of (a) CPCM, (b) CMPCM-2, and (c) CMPCM-4. High-resolution XPS C 1s spectra of (d) CPCM, (e) CMPCM-2, and (f) CMPCM-4. N 1s spectra of (g) CMPCM-2 and (h) CMPCM-4. And (i) 0 1s spectra of CMPCM-4. 

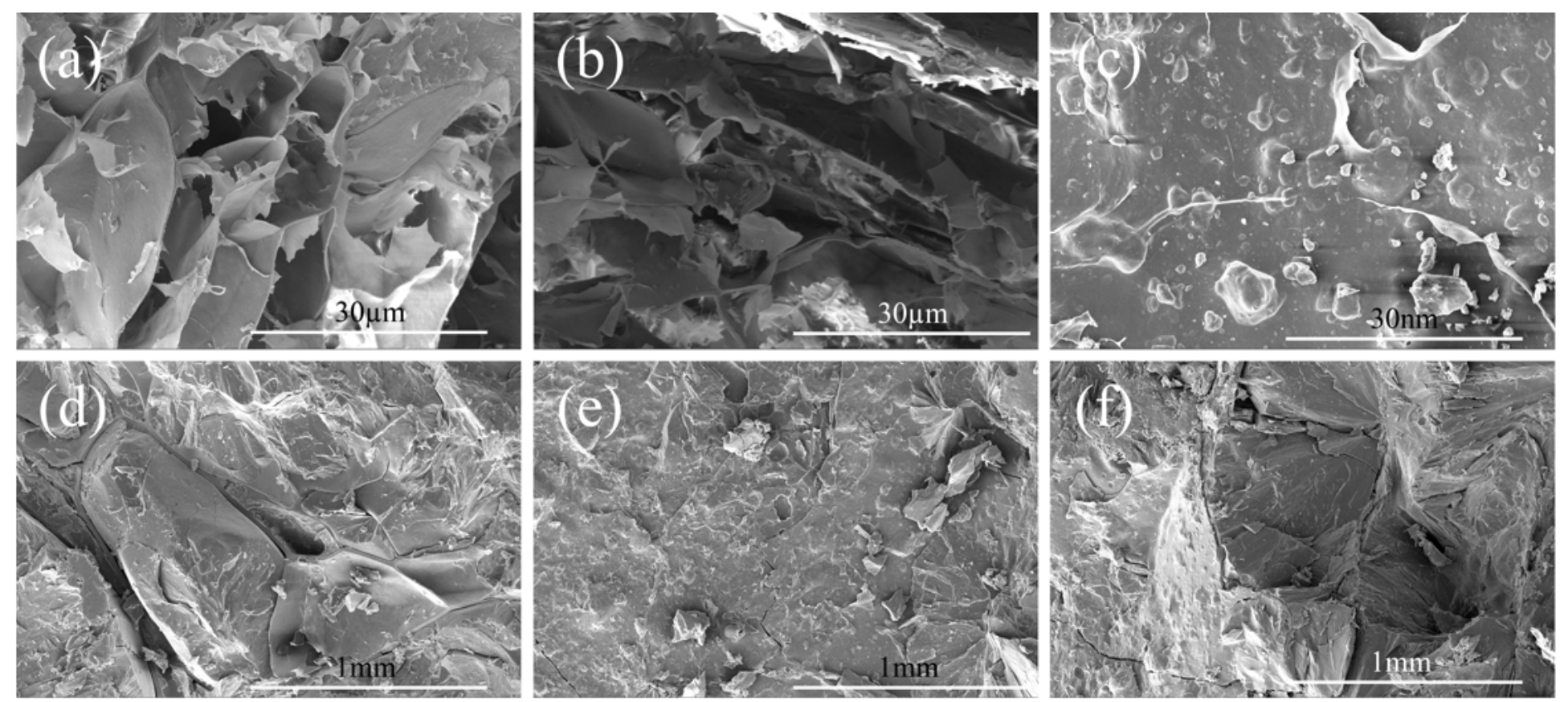

Figure 3

The SEM image of: (a) CA, (b) CMA-2, (C) CMA-4, (d) CPCM, (e) CMPCM-2, and (f) CMPCM-4.

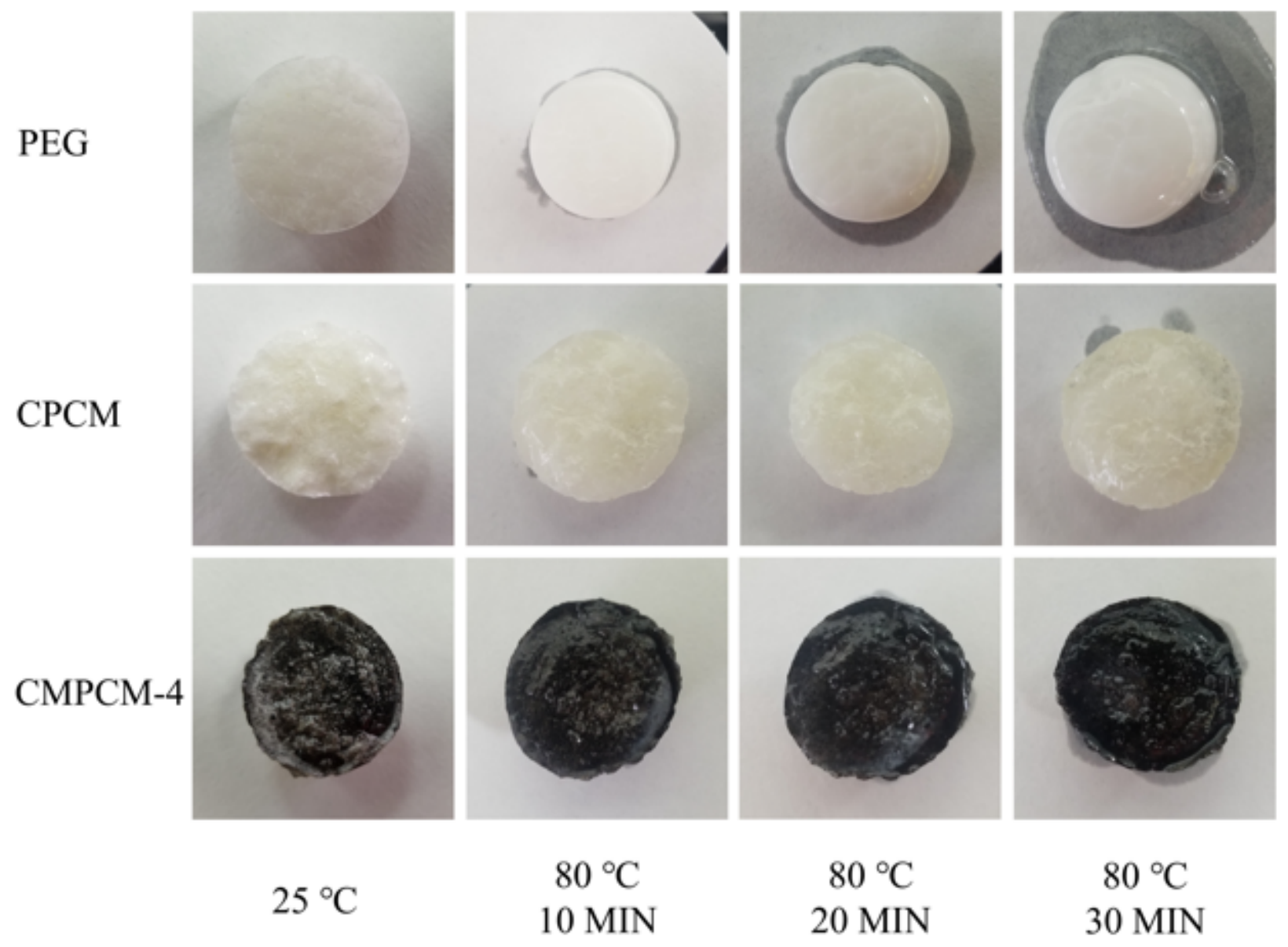

Figure 4 
Digital photographs of PEG, CPCM, and CMPCM-4 at $25^{\circ} \mathrm{C}$ and $80^{\circ} \mathrm{C}$.
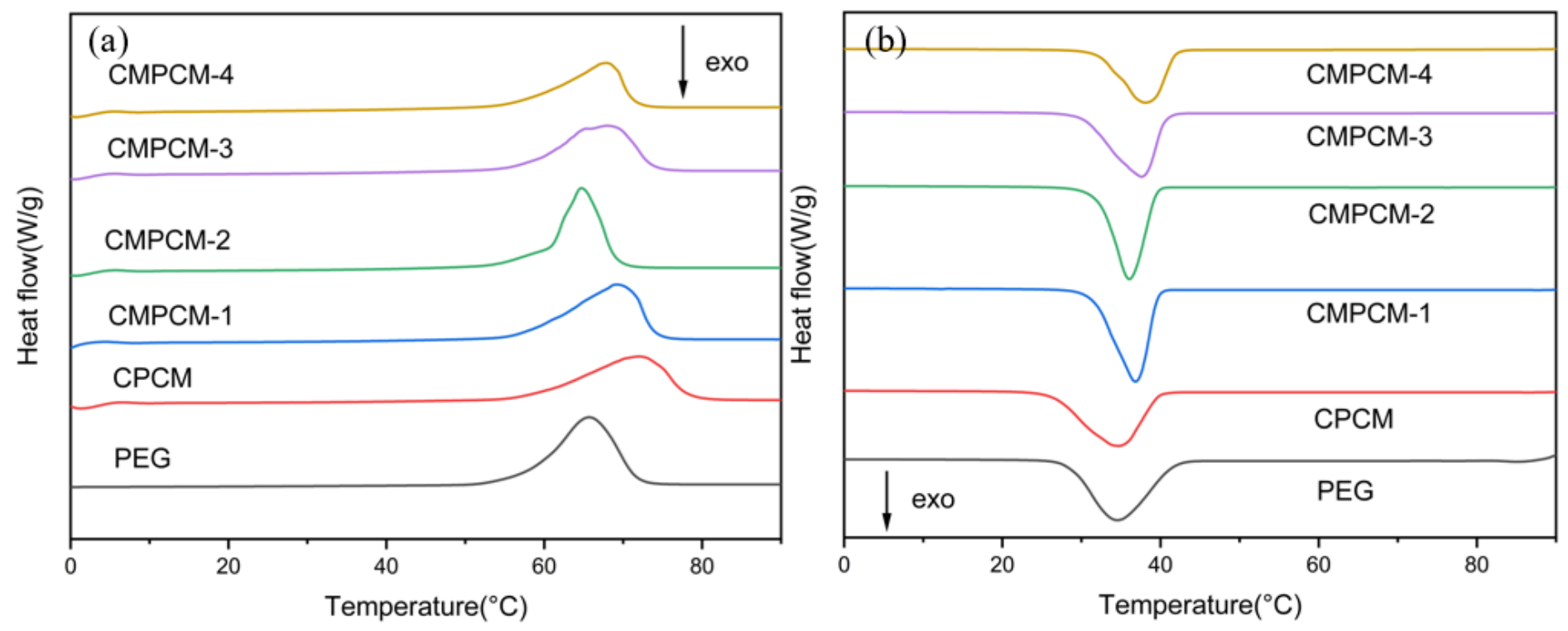

Figure 5

DSC heating (a) and cooling (b) curves of CPCM, CMPCMs, and PEG.

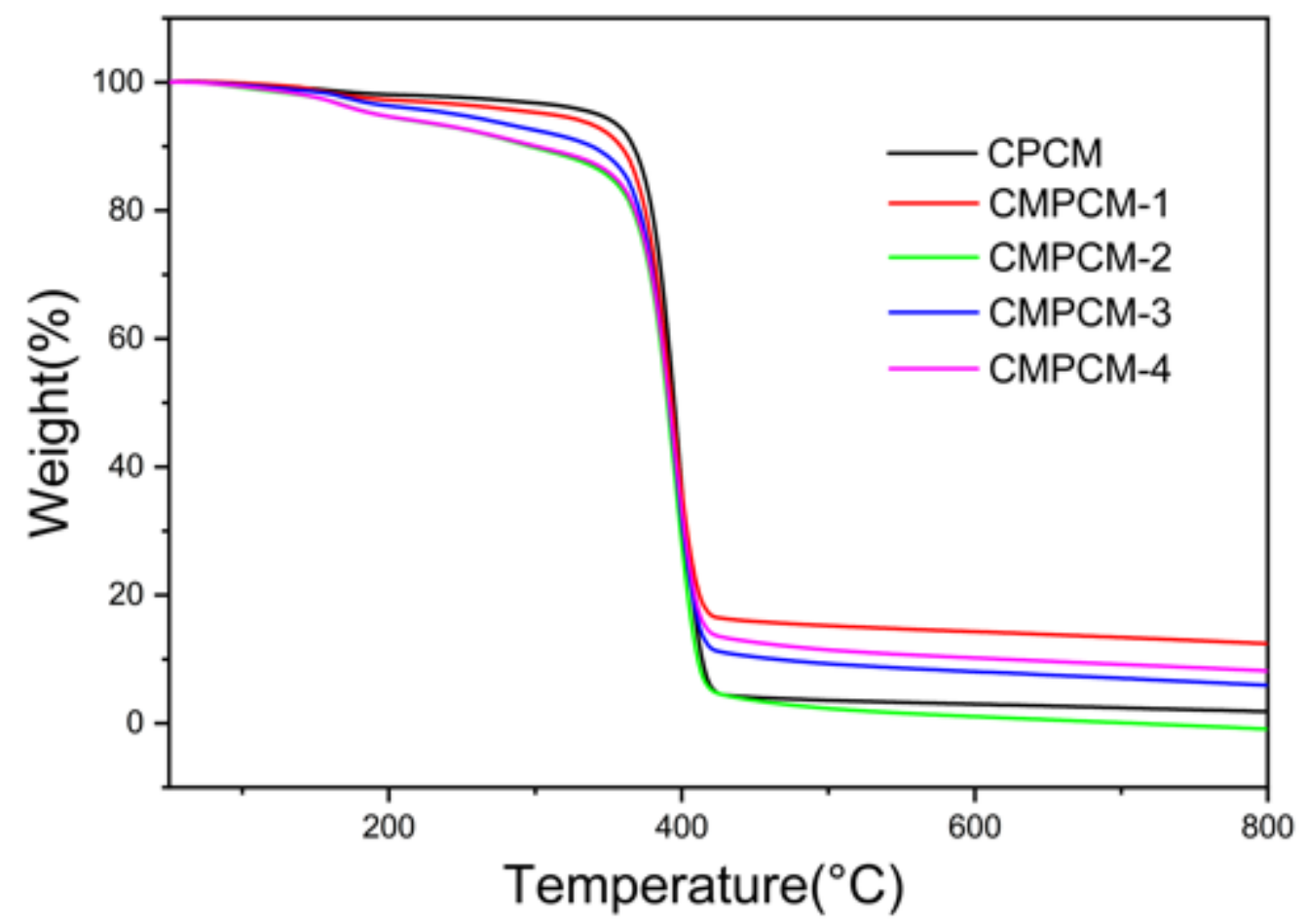

Figure 6

TG curves of CPCM, CMPCMs, and PEG. 


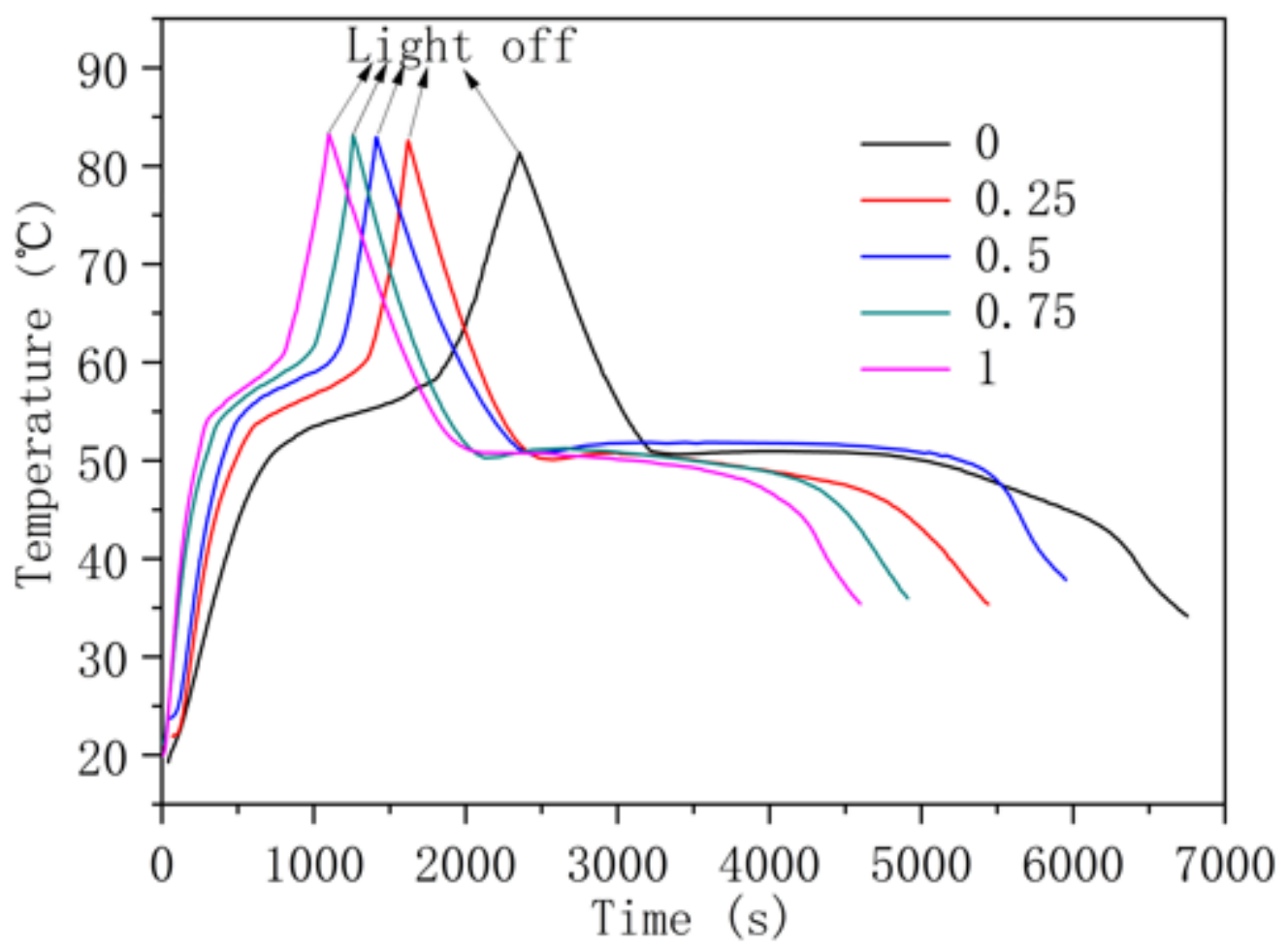

Figure 7

Temperature variation curves of CPCM and CMPCMs under light irradiation of $250 \mathrm{~mW} \mathrm{~cm}-2$.

\section{Supplementary Files}

This is a list of supplementary files associated with this preprint. Click to download.

- scheme1.png

- scheme2.png 